\title{
Mapping the Emission Location of the Crab Pulsar's Giant Pulses
}

\section{Robert Main and Marten H. van Kerkwijk $\dagger$}

Department of Astronomy \& Astrophysics, University of Toronto, 50 Saint George Street, Toronto, ON, M5S 3H4, Canada

email: main,mhvk@astro.utoronto.ca

\begin{abstract}
The Crab pulsar has a striking radio profile, dominated by two pulse components (the main pulse and interpulse) which are comprised of giant pulses. These pulses are randomly occurring, they extend to extremely high flux densities, and are closely aligned with emission across the entire electromagnetic spectrum. The Crab, like many pulsars, exhibits scintillation - a pattern in frequency and time arising from interfering scattered images. The pattern varies with location, with the physical scale over which it changes by order unity corresponding to the spatial resolution of the scattering surface. For the Crab, the scattering is in the nebula and the estimated spatial resolution is of order the light cylinder radius. Comparing scintillation spectra of the two components, we infer a difference in physical location of the same order.
\end{abstract}

Keywords. pulsars: individual (Crab), scattering, techniques: interferometric

The results presented in this contribution are being published, as Main et al. (2017).

\section{Reference}

Main, R., van Kerkwijk, M. H., Pen, U.-L., Rudnitskii, A. G., Popov, M. V., Soglasnov, V. A., \& Lyutikov, M., 2017, ApJL, submitted (arXiv:1709.09179) 